\title{
Dipole Straylight Contamination and Low Multipoles
}

\author{
Alessandro Gruppuso, Carlo Burigana \\ INAF-IASF Bologna, via Gobetti 101, I-40129 Bologna, Italy \\ E-mail: gruppusodiasfbo.inaf.it buriganadiasfbo.inaf.it
}

\section{Fabio Finelli}

INAF-IASF Bologna, via Gobetti 101, I-40129 Bologna, Italy

and INAF-OAB, via Ranzani 1, I-40127 Bologna, Italy

E-mail: finelli@iasfbo.inaf.it

\begin{abstract}
Kinematic dipole signal originates from the motion of the Sun with respect to the CMB rest frame. It is a remarkable signal, two orders of magnitude larger than the observed pattern of CMB anisotropies. Therefore the dipole subtraction from the main beam is of primary importance. But what happens to the dipole signal entering the main spillover (i.e. the most relevant far antenna pattern feature, responsible for the bulk of the straylight effect)? We present here an analytical and statistical study of this systematic effect for a spinning space-mission like PLANCK *
\end{abstract}

\footnotetext{
PACS: $98.70 . \mathrm{VC}, 07.87 .+\mathrm{v}, 95.55 .-\mathrm{n}, 02.50 . \mathrm{Fz}$
}

$C M B$ and Physics of the Early Universe

20-22 April 2006

Ischia, Italy

\footnotetext{
*This work has been done in the framework of the PLANCK LFI activities.
} 


\section{Analytical Description}

The convolution $I$ of the dipole signal with the main spillover ${ }^{1}$ can be written as:

$$
I=\int d \Omega T_{1 m} Y_{1}^{m}(\theta, \varphi) B_{S L}(\theta, \varphi),
$$

where $d \Omega=d \theta \sin \theta d \varphi$ is the solid angle element, $\theta \in[0, \pi]$ is the colatitude, $\varphi \in[0,2 \pi$ [ is the longitude, and where the sum on $m$ over $-1,0,1$ is understood. $T_{1 m}$ are the coefficients of the expansion of the dipole ${ }^{2}$ on the spherical harmonics basis $Y_{1}^{m}(\theta, \varphi)$ and $B_{S L}(\theta, \varphi)$ is the beam response representing the shape of the main spillover in the $(\theta, \varphi)$-plane. In this notation $B_{S L}$ is normalized to the whole beam integrated response, dominated by the contribution of the main beam $\int_{4 \pi} d \Omega B \simeq \int_{\text {main beam }} d \Omega B \simeq 2 \pi \sigma_{b}^{2}$ where $\sigma_{b}=\mathrm{FWHM} / \sqrt{8 \ln 2}$. Notice that $I$ is a function that depends on the istantaneous orientation of the main spillover.

Of course eq. (1.1) is general and exact. Any specific approximation of $B_{S L}$ (inspired by experiments) will introduce a certain degree of uncertainty with respect to the real case. Our current approximation for $B_{S L}$ is the Top Hat [1, 2]]:

$$
B_{S L}(\theta, \varphi)=f_{S L} \Delta\left(\theta, \theta_{m s}-\Delta_{\theta<}, \theta_{m s}+\Delta_{\theta>}\right) \Delta\left(\varphi, \varphi_{m s}-\Delta_{\varphi<}, \varphi_{m s}+\Delta_{\varphi>}\right)
$$

where $f_{S L}=p / \Omega_{m s}$ is a constant ( $p$ is the ratio between the power entering the main spillover and the power entering the main beam, $\Omega_{m s}$ is the solid angle subtented by the main spillover) and $\Delta(a, b, c)=S(a-b)-S(a-c)$ with $S(x)$ representing the step function (or Heavyside function) that takes the value 1 for $x \geq 0$ and the value 0 otherwise. Eq. (1.2) is just an asymmetric rectangular box, in the $(\theta, \varphi)$-plane, centered around the point $\left(\theta_{m s}, \varphi_{m s}\right)$ and with sides of length $\Delta_{\theta>}+\Delta_{\theta<}$ and $\Delta_{\varphi>}+\Delta_{\varphi<}$. Notice that the point $\left(\theta_{m s}, \varphi_{m s}\right)$ is the pointing direction of the main spillover. With $\varepsilon$ and $\delta$ implicitely defined by $\Delta_{\theta>}=\Delta_{\theta}+\delta$ and $\Delta_{\varphi>}=\Delta_{\varphi}+\varepsilon$ where $\Delta_{\theta<}=\Delta_{\theta}$ and $\Delta_{\varphi<}=\Delta_{\varphi}$, we obtain the final expression for the convolution (1.1):

$$
\begin{aligned}
& I^{T H} / f_{S L}=\frac{T_{10}}{2} \sqrt{\frac{3}{4 \pi}} \sin \left(\delta+2 \Delta_{\theta}\right) \sin \left(\delta+2 \theta_{m s}\right)\left(2 \Delta_{\varphi}+\varepsilon\right)- \\
& 4 \sqrt{\frac{3}{8 \pi}}\left(\Delta_{\theta}+\frac{\delta}{2}-\cos \left(\delta+2 \theta_{m s}\right) \frac{\sin \left(\delta+2 \Delta_{\theta}\right)}{2}\right) \operatorname{Re}\left[T_{11} e^{i\left(\varphi_{m s}+\varepsilon / 2\right)}\right] \sin \left(\Delta_{\varphi}+\frac{\varepsilon}{2}\right)
\end{aligned}
$$

where the label ${ }^{T H}$ stands for Top Hat.

During the rotation of the main beam (assumed, for simplicity, at $90^{\circ}$ from the spin axis) in each scan circle, the main spillover, if not lying on the spin axis, draws a cone. We define $\alpha$ the angle between the main spillover direction and the spin axis ${ }^{3}$. The total signal received by the satellite, is the sum of the two following terms: $T(\theta, \varphi)=T_{M B}(\theta, \varphi)+I_{S L}(\theta, \varphi)$ where $T_{M B}$ is the signal entering the main beam where the dipole has been subtracted away, whereas $I_{S L}$ is the signal

\footnotetext{
${ }^{1}$ It is the main feature of the antenna pattern far from the main beam.

${ }^{2} \mathrm{We}$ use the symbol $T_{\ell m}$ in order to make clear that the dimensionality is a temperature $\left({ }^{\circ} \mathrm{K}\right)$. Moreover we work in a frame centered on the spacecraft with axes pointing fi xed (far away) stars. In this frame the dipole vector is constant while the direction of the main spillover varies (it rotates of $2 \pi$ per year in a nominal scanning strategy).

${ }^{3}$ For simplicity we assume here the main spillover centre located on the plane defi ned by the spin axis and the beam centre direction.
} 
due to the dipole entering the main spillover. In the case of a PLANCK nominal scanning strategy [3], for the first (upper signs) and the second (lower signs) survey, $I_{S L}$ is given by

$$
I_{S L}^{(I / I I)}(\theta, \varphi)=\left\{\begin{array}{c}
I^{T H}(\pi / 2-\alpha \cos \theta, \varphi \pm \pi / 2 \mp \alpha \sin \theta) \text { for } 0<\varphi<\pi, \\
I^{T H}(\pi / 2-\alpha \cos \theta, \varphi \mp \pi / 2 \pm \alpha \sin \theta) \text { for } \pi<\varphi<2 \pi .
\end{array}\right.
$$

The shift in the definition of the arguments in $I_{S L}$ (during either the first or the second survey) comes from the fact that when the main beam rotates from North to South the main spillover is shifted of $-\pi / 2$ plus a small correction proportional to $\alpha$ (due to the non perfect alignment of the main spillover with the spin axis), while when the main beam rotates from South to North the main spillover is shifted of $+\pi / 2$ minus a small correction again proportional to $\alpha$. Notice that $(\theta, \varphi)$ is the direction of pointing of the main beam.

\section{General Analytical Results}

We set now $T_{M B}=0$ and take into account only the straylight signal,

$$
T_{\ell m}^{S L}=\int d \Omega I_{S L}(\theta, \varphi) Y_{\ell m}^{\star}(\theta, \varphi)
$$

because we want to analyze only the map due to the Dipole Straylight Contamination (DSC). Some general properties can be found $\llbracket$ $\rrbracket$, once performed an expansion for small $\alpha$ : $T_{\ell m}^{S L}=$ $T_{\ell m}^{(0)}+\alpha T_{\ell m}^{(1)}+\mathscr{O}\left(\alpha^{2}\right)$. In particular for an odd number of surveys to the leading order in $\alpha$ only the even multipoles survive [i.e. $T_{\ell m}^{(0)} \neq 0$ for even $\ell$ (and $m$ )] while to the linear order only the $\ell=1$ term turns on. For an even number of surveys DSC vanishes except for $\ell=1$, $\hat{T}_{\ell m}^{S L}=\left(T_{\ell m}^{(1 s t)}+T_{\ell m}^{(2 n d)}\right) / 2=0+\alpha T_{\ell m}^{(1)}+\mathscr{O}\left(\alpha^{2}\right)$. These qualitative features have been tested and extended by numerical simulations [ [ 4 . As an example, we show the analytical expressions derived for the dipole

$$
\begin{array}{r}
T_{10}^{S L}=2 \sqrt{4 \pi / 3} c_{1} \alpha \\
T_{1 \pm 1}^{S L}=(1 / 2) \sqrt{8 \pi / 3}\left( \pm d_{1}+i d_{2}\right)\left(c_{2}+c_{3}\right) \alpha
\end{array}
$$

and the non vanishing coefficients for the quadrupole

$$
T_{2 \pm 2}^{S L}=-(4 / 3)^{2} \sqrt{15 / 32 \pi}\left(d_{1} \pm 2 i d_{2}\right)\left(c_{2}+c_{3}\right),
$$

where $c_{1}=\sqrt{3 / 4 \pi} f_{S L} \Delta \sin (2 \Delta) T_{10}, c_{2}=4 \sqrt{3 / 8 \pi} f_{S L} \Delta, c_{3}=4 \sqrt{3 / 8 \pi} f_{S L} \sin (2 \Delta) / 2, d_{1}=$ $\sin \Delta \operatorname{Re}\left[T_{11}\right], d_{2}=\sin \Delta \operatorname{Im}\left[T_{11}\right]$, with $\Delta_{\theta}=\Delta_{\varphi}=\Delta$ and $\varepsilon=\delta=0$.

\section{Dipole and Quadrupole Analysis}

Section 2 shows that when the main spillover direction is different from the spin axis (i.e. $\alpha \neq 0$ ) then the Dipole itself is modified. This has an impact on the calibration with amplitude related to the value of $p$. For $\Delta_{\theta}=\Delta_{\varphi}=\Delta=\pi / 10, \alpha=\pi / 18$ and $p=1 / 100$, we estimate that $T_{10}^{S L}=-2.1 \mu K, T_{11}^{S L}=(7.7 \pm 1.0 i) \mu K$. This leads to $C_{1}^{S L}=44.5 \mu K^{2}$. 

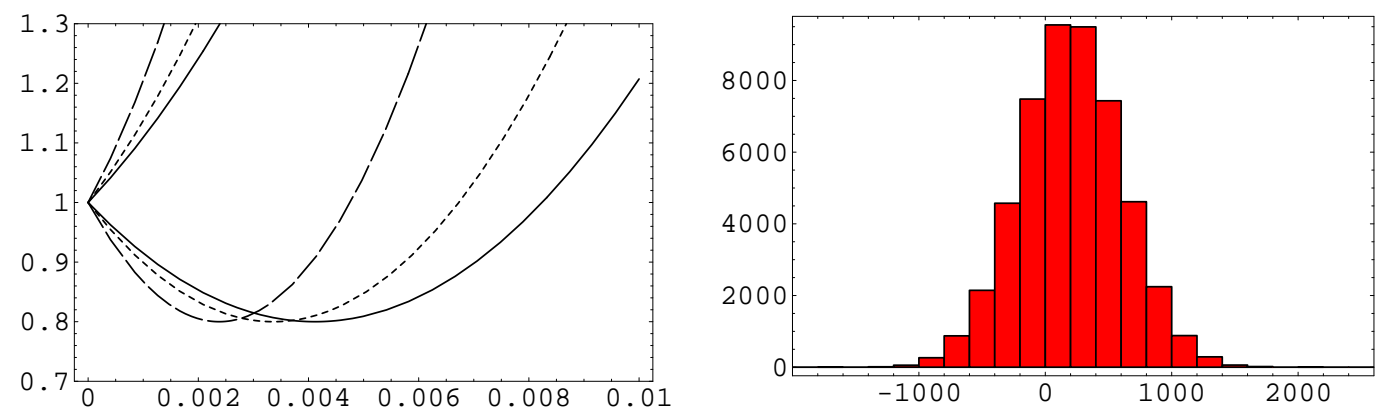

Figure 1: Left Panel: $y=y\left(F_{S L}\right)$ with $B>0$ (lower curves). Solid line is for $C_{2}^{S K Y}=1500 \mu K^{2}$, dotted line is for $C_{2}^{S K Y}=1000 \mu K^{2}$ and dashed line is for $C_{2}^{S K Y}=500 \mu K^{2}$. We report also the case of $B<0$ (upper curves) which does not imply a quadrupole decreasing but only a quadrupole increasing. Right Panel: Distribution of the DSC, i.e. $C_{2}-C_{2}^{S K Y}$. See also the text.

The next multipole that is modified by DSC is the Quadrupole. We can write the observed quadrupole $C_{2}$ as $C_{2}\left(F_{S L}\right)=C_{2}^{S K Y}-\frac{4}{5} B F_{S L}+\frac{2}{5} A F_{S L}^{2}$, where $C_{2}^{S K Y}$ is the intrinsic quadrupole (that would be observed if the DSC would be absent), $B \equiv \operatorname{Re}\left[T_{11}\right] \operatorname{Re}\left[T_{22}^{S K Y}\right]+2 \operatorname{Im}\left[T_{11}\right] \operatorname{Im}\left[T_{22}^{S K Y}\right]$, $A \equiv\left[\operatorname{Re}\left[T_{11}\right]\right]^{2}+4\left[\operatorname{Im}\left[T_{11}\right]\right]^{2}$ and $F_{S L}=(\sqrt{5} p / 3 \pi)[1+\cos \Delta \sin \Delta / \Delta]$. Depending on the sign of $B$ (or in other words on the sign of the real and the imaginary part of $T_{22}^{S K Y}$ ) we have two different kinds of behaviour for the observed quadrupole in terms of $F_{S L}$. It can only increase for $B<0$ but it can also decrease for $B>0$. In the left panel of Fig. 1 the two branches are plotted for the function $y\left(F_{S L}\right)=C_{2}\left(F_{S L}\right) / C_{2}^{S K Y}$ where, as an example, it has been set $\operatorname{Re}\left[T_{22}^{S K Y}\right] \sim \pm\left(C_{2}^{S K Y} / 2\right)^{1 / 2}$ and where it has been considered $\operatorname{Im}\left[T_{11}\right] \ll \operatorname{Re}\left[T_{11}\right]$. In this regime $\operatorname{Im}\left[T_{22}^{S K Y}\right]$ does not enter in the estimate.

Since the behaviour of the observed quadrupole depends on the sky realization $\left(T_{22}^{S K Y}\right)$, we present a statistical analysis aimed at the computation of the probability of increasing or decreasing of $C_{2}$. We have made 50000 Gaussian distributed extractions of $T_{2 m}^{S K Y}$ such that on average $C_{2}^{S K Y} \sim 1000 \mu K^{2}$ and we have computed the distribution for the observed quadrupole $C_{2}$ and for the intrinsic quadrupole. In the right panel of Fig. 1 we show the plot of the distribution for the pure DSC (i.e. the difference $C_{2}-C_{2}^{S K Y}$ ). We note that the distribution of DSC has a Gaussian profile and we compute its mean $\left(202.3 \mu K^{2}\right)$ and standard deviation $\left(404.3 \mu K^{2}\right)$. We conclude that the $C_{2}$ probability of increasing (decreasing) is $69 \%$ (31\%).

\section{References}

[1] A. Gruppuso, C. Burigana and F. Finelli, On the dipole straylight contamination in PLANCK-like CMB anisotropy missions: an analytical approach, Internal Report IASF-BO 405/2004.

[2] A. Gruppuso, C. Burigana and F. Finelli, An analytical approach to low multipole effects from the dipole straylight contamination in PLANCK-like CMB anisotropy missions, Internal Report IASF-BO 416/2005.

[3] X. Dupac and J. Tauber, Scanning strategy for mapping the Cosmic Microwave Background anisotropies with Planck, A\&A 430, 363-371 (2005) [ast ro-ph/ 0409405 ].

[4] C. Burigana, A. Gruppuso and F. Finelli, On the dipole straylight contamination in spinning space missions dedicated to CMB anisotropy, accepted for publication in MNRAS [ast ro-ph/0607506]. 